\title{
Real Time Fluoroscopy Guided Extraction of an Impacted Oesophageal Foreign Body: A Case Report
}

\section{Ukamaka Nwankwo*}

Department of ENT, Nigerian Navy Reference Hospital, Lagos, Nigeria

*Corresponding Author: Ukamaka Nwankwo, Department of ENT, Nigerian Navy

Reference Hospital, Lagos, Nigeria.
Received: July 08, 2021

Published: July 22, 2021

C All rights are reserved by Ukamaka

Nwankwo.

\section{Abstract}

The incidence of children swallowing foreign bodies or items is high because of their exploratory tendencies. Sharp or large foreign items can get impacted in the oesophagus with associated complications. Duration of impaction and poor visualization of the foreign item may make the extraction process difficult.

This is a case report of the management of a 9-year-old boy with 6 days history of oesophageal foreign item impaction. He had emergency rigid oesophagoscopy with real time fluoroscopy guided extraction of the foreign body. This report highlights the advantage of using real time fluoroscopy in the extraction of impacted, metallic, foreign items.

Keywords: Foreign Bodies; Real Time Fluoroscopy; Oesophagus; Impacted

\section{Introduction}

The incidence of swallowing foreign bodies or items irrespective of size, shape or constituency is high in children [1,2]. It could occur unintentionally especially while playing or when startled. These foreign items can get impacted in the oesophagus if they are large or have irregular configurations [3] and the cervical part of the oesophagus is the most frequent site of impaction [4]. The impaction of these foreign items in the oesophagus is often associated with complications which could be life threatening and therefore regarded as emergencies. Obstruction of the oesophageal lumen, perforation of the walls of the oesophagus leading to dislodgement of the foreign item into another cavity, bleeding or mediastinitis are complications associated with impaction $[5,6]$. These complications determine the presentation of the patient and may become worse if there is delayed presentation to a medical facility. Affected patients may present with difficulty in swallowing, painful swallowing, dribbling of saliva, chest pain [7] and foreign body sensation in the oesophagus.

The method of extraction of impacted foreign items and the expedition of this process depends on the following factors: size, type of foreign item, site of impaction, duration of impaction, associated complications and surgeon's preference $[1,8]$. The likelihood of oesophageal wall perforation is increased fourteen times if foreign item impaction lasts more than twenty-four hours [4]. Therefore, the main goal of treatment is the urgent extraction of the foreign body or item to prevent, arrest or reverse complications and also to improve success rate of removal. Oesophagoscopy has been advocated for the removal of these foreign items due to its more than ninety five percent success rate [9] and should be done as soon as possible with expertise and good visualization of the foreign item.

This case presentation describes the surgical extraction of a sharp, foreign item, impacted in the thoracic part of the oesophagus in a 9-year-old boy under real time fluoroscopy guidance. A small number of studies have reported real time fluoroscopy guided removal of impacted, metallic, oesophageal foreign bodies.

\section{Case Study}

A 9-year-old boy presented at the emergency department with 6 days history of accidental swallowing of a metallic shoe accessory. This occurred while he was playing with the accessory in his 
mouth. There was associated dysphagia both to food and fluids, painful swallowing and severe retrosternal pain. There was no cough, dyspnoea, hoarseness or aspiration. He was forced at home to push down the metallic, shoe accessory with food morsels hopefully into his stomach but this worsened the dysphagia and chest pain. He was subsequently taken to a general hospital but was referred to this facility due to unavailability of required equipment for extraction.

He was noted to be in painful distress, clenching his chest repeatedly, weak and dehydrated. Vital signs and chest examination were normal. Antero- posterior and lateral chest radiographs displayed a radio opaque shoe accessory in the thoracic part of the oesophagus at T3-T4 level. A diagnosis of oesophageal foreign body impaction was made.

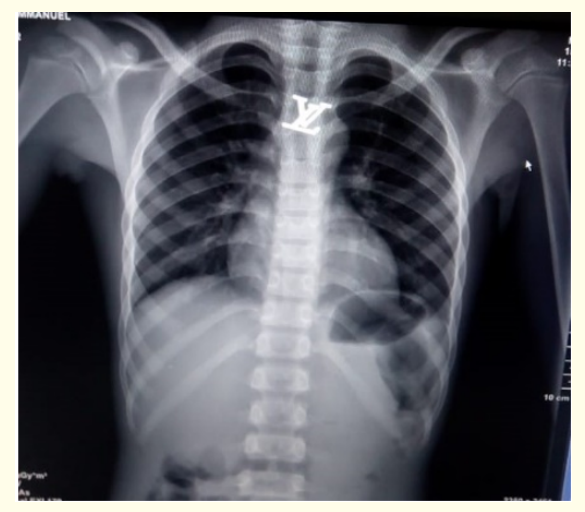

Figure 1: Chest radiograph (anterior-posterior view).

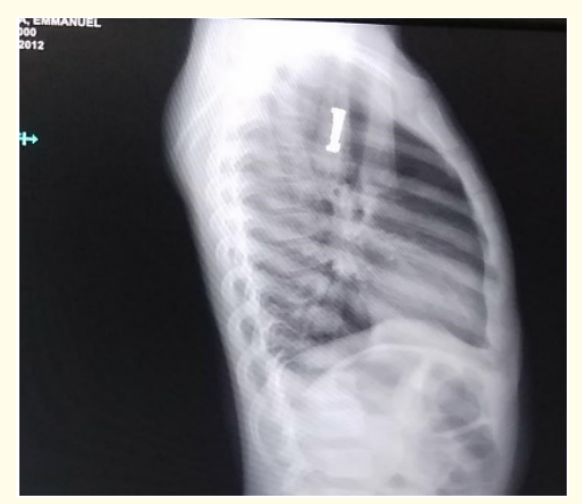

Figure 2: Chest radiograph (lateral view).
He had emergency, rigid oesophagoscopy with intraoperative findings of food particles and marked oedema of the oesophageal mucosa at the site of impaction. Only the tip of the foreign item was visualized. Multiple attempts at dislodgement and extraction of the shoe accessory proved abortive. It was finally removed with difficulty using real time fluoroscopy guidance with better visualization of the foreign item, grasping forceps and the process of extraction of the impacted foreign body.

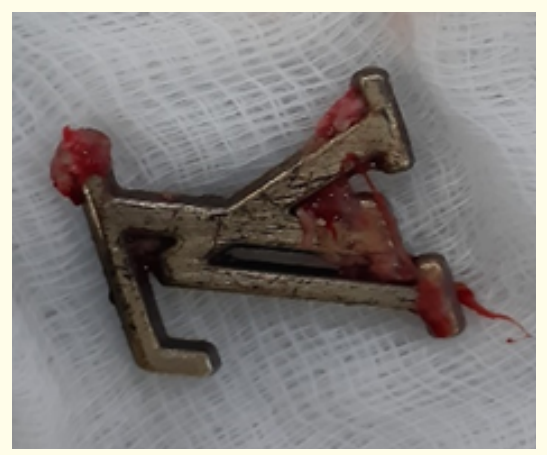

Figure 3: Metallic shoe accessory post extraction.

Examination of the oesophagus post extraction revealed mucosal injury which necessitated the insertion of a nasogastric tube. Post operatively he was placed on intravenous antibiotics, analgesics and fluid. He was fed via the nasogastric tube on the $4^{\text {th }}$ postoperative day and oral feeds commenced on the $6^{\text {th }}$ post-operative day following normal chest radiograph finding. He was then discharged.

Follow up clinical visits were unremarkable.

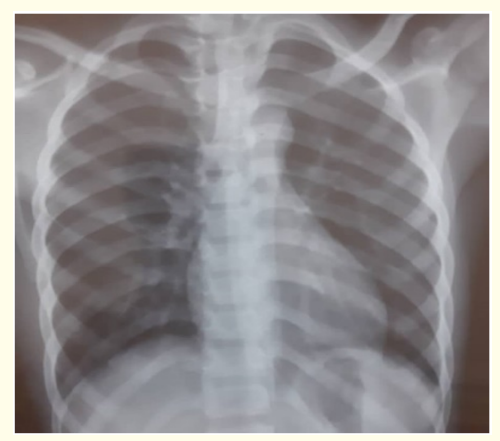

Figure 4: Post extraction chest radiograph. 


\section{Discussion}

The incidence of children unintentionally swallowing foreign items is high $[8,10]$ because they are naturally curious and place these items in their mouths to examine them. They also place these items in their mouth as part of childhood games. This tends to occur more frequently in male children because of their exploratory and playful tendencies $[11,12]$. Most times, these items are swallowed without any harm and passed out in stool.

Sharp or large items often gets stuck in the oesophagus and this occurs more in regions of anatomical constrictions or constrictions due to a disease process [13]. The anatomical sites of constriction in the oesophagus include the upper oesophageal sphincter at C6, the region of the aortic crossing at $\mathrm{T} 4$ and the lower oesophageal sphincter [13]. The metallic shoe accessory swallowed in this case study was impacted in the T3-T4 region of the oesophagus which is the site of anatomical constriction of the oesophagus caused by the crossing of the aorta.

The process of foreign body extraction especially of sharp objects is determined by the duration of ingestion, associated complications, available equipment and expertise of the clinician. The item should be clearly visualized as blind procedures can lead to failed extraction attempts, dislodgement distally or into another cavity. This child had emergency rigid oesophagoscopy due to the fear of the complications associated with prolonged, sharp foreign item impaction which in this case was a metallic Louis Vuitton shoe accessory. The impaction was further worsened by the repeated attempts at pushing the shoe accessory down the oesophagus with hard food morsels prior to presentation. This made extraction difficult as food particles in the oesophagus prevented clear visualization of the foreign item and the foreign item was also well embedded in the mucosa. Real time fluoroscopy made extraction of the foreign item successful.

Fluoroscopy is an imaging modality that displays uninterrupted $\mathrm{x}$-ray images on a screen. The series of consecutive x-ray images produced during fluoroscopy is so fast that the eye visualizes these images as a continuous or uninterrupted flow (real time) [14]. This is the advantage that it has over conventional radiographs that display motionless images. Similar to radiographs, radio opaque objects and instruments are easily seen. That was why fluoroscopy was beneficial in this case as both the foreign item and instruments of extraction were radio opaque. This made the dislodgement of the foreign item effortless. The foreign item was also monitored as it was extracted. Therefore, the advantage of fluoroscopy in metallic foreign body extraction includes proper visualization of the metallic item and the extraction process without or with limited complications.

\section{Conclusion}

Real time fluoroscopy is beneficial in the extraction of impacted, metallic foreign bodies in the oesophagus as it provides a clear image of the foreign item and guides the surgeon in the extraction process. It also prevents or reduces the complications associated with the extraction of sharp foreign bodies. It should be considered when available for the safe removal of an impacted oesophageal foreign item.

\section{Bibliography}

1. Oguntoye 00., et al. "Removal of an impacted foreign body from the upper airway with a gastroscope in a tertiary hospital in south-west Nigeria- A case report". Gastroenterology, Hepatology and Digestive Disorders 3.1 (2020): 1-5.

2. Salih A., et al. "Airway foreign bodies. A critical review for a common pediatric emergency". World Journal of Emergency Medicine 7 (2016): 5-12.

3. Afolabi OA., et al. "Re-emergence of coin in Nigerian currency: Implication in medical practice". The Internet Journal of Otorhinolaryngology 9.1 (2009).

4. Riva CG., et al. "Unusual foreign body impacted in the upper oesophagus: original technique for transoral extraction". BMJ Case Reports (2018).

5. Bonavina, L., et al. "Thoracoscopic removal of dental prosthesis impacted in the upper thoracic esophagus". World Journal of Emergency Surgery 9 (2014): 5.

6. Dray X and Cattan P. "Foreign bodies and caustic lesions". Best Practice and Research Clinical Gastroenterology 27 (2013): 679-689.

7. Ashraf 0. "Foreign body in the esophagus. A review". Sao Paulo Medical Journal 126 (2006): 346-349.

8. Birk M., et al. "Removal of foreign bodies in the upper gastrointestinal tract in adults: European Society of Gastrointestinal Endoscopy (ESGE) Clinical Guideline”. Endoscopy 48 (2016): 1-8. 
9. Wyllie R. "Foreign bodies in the gastrointestinal tract". Current Opinion in Pediatrics 18.5 (2006): 563-564.

10. Guven DG., et al. "Fluoroscopy guided removal of a metallic foreign body in the neck: a case report”. Konulrap Tip Dergisi 5.1 (2013): 42-45.

11. Gupta P and Jain AK. "Foreign bodies in upper aero-digestive tract: A clinical study". International Journal of Research in Medical Sciences 2 (2014): 886-891.

12. Ibekwe MU., et al. "Swallowed foreign bodies in the University of Port harcourt Teaching Hospital; Profile and challenges in management". IAR Journal of Medical Sciences 2.2 (2021): 181187.

13. Conners GP and Mohseni M. "Pediatric Foreign Body Ingestion". In: StatPearls [Internet]. Treasure Island (FL). Stat Pearls Publishing (2020).

14. Gingold E. Modern fluoroscopy imaging systems (2014).

Volume 3 Issue 8 August 2021

(C) All rights are reserved by Ukamaka Nwankwo. 